\title{
FRIENDS OF RADIOCARBON
}

RADIOCARBON recognizes the following for their generous contributions beyond their regular subscription orders:

\section{INDIVIDUALS}

Berit Andersen, Umea, Sweden L. D. Arnold, Vegreville, Alberta, Canada Georges Bonani, Zürich, Switzerland J. Benedict, Ward, Colorado Gordon T. Cook, East Kilbride, Scotland Douglas J. Donahue, Tucson, Arizona P. Q. Dresser, Swansea, Wales Jacques Evin, Lyon, France Philippe Fortin, Lyon, France Svend Funder, Copenhagen, Denmark D. D. Harkness, East Kilbride, Scotland Ede Hertelendi, Debrecen, Hungary Achim Hiller, Leipzig, Germany Salvatore Improta, Rome, Italy A. J. T. Jull, Tucson, Arizona Robert M. Kalin, Belfast, Northern Ireland Kunihiko Kigoshi, Tokyo

Peter I. Kuniholm, Ithaca, New York Joan S. Mestres, Barcelona, Spain H. N. Michael, Philadelphia, Pennsylvania Jaan-Mati Punning, Tallinn, Estonia Irving Rouse, New Haven, Connecticut E. Marian Scott, Glasgow, Scotland Jerry J. Stipp, Coral Gables, Florida R. E. Taylor, Riverside, California David Hurst Thomas, New York, New York J. Peter Thurmond, Cheyenne, Oklahoma Susan E. Trumbore, Irvine, California Murry A. Tamers, Coral Gables, Florida Johannes van der Plicht, Groningen John S. Vogel, Livermore, California Osamu Yamada, Kyoto, Japan

\section{LABORATORIES AND INSTITUTIONS}

Arkiologiska Inst., Umea Universitet, Umea, Sweden

Arizona-NSF Accelerator Facility, Tucson Beta Analytic, Inc., Coral Gables, Florida Centre de Datation par le RadioCarbone, Lyon, France

Centre for Isotope Research, Groningen High Voltage Engineering Europa B.V., Amersfoort, The Netherlands Isotopes Laboratory, Waterloo, Canada Laboratori de Datacio per Radiocarboni, Barcelona, Spain

Laboratory of Isotope Geochemistry, Tucson Naturhistoriska Riksmuseet, Stockholm NERC Laboratory, East Kilbride, Scotland ${ }^{14} \mathrm{C}$ Lab, Gakushuin University, Tokyo ${ }^{14} \mathrm{C}$ Lab, University of Texas at Austin Radiocarbon Laboratory, Swansea, Wales Rome ${ }^{14} \mathrm{C}$ Laboratory-Fisica, Rome, Italy SURRC, East Kilbride, Scotland UCR, Riverside, California 